\title{
Positivity, space scale and convergence towards the equilibrium
}

\author{
Jean-Luc Gouzé \\ INRIA \\ BP 93 \\ 06902 Sophia-Antipolis Cedex \\ France \\ email gouze@sophia.inria.fr \\ fax (33) 93657765
}

\begin{abstract}
All the variables of the biological models are positive. We examine the constraints that one has to put on the model to verify such a property. For a linear (differential) model, it implies that the equilibrium is stable. For the n-dimensional Lotka-Volterra models, the positive orthant is invariant, but we impose stronger constraints to prevent the solutions to go upon or below some given thresholds, that define the space scale of the model. Then we show that, under reasonable hypothesis, this implies the global convergence towards the equilibrium.
\end{abstract}

Keywords: positivity, Lotka-Volterra models, global convergence.

\section{Introduction}

For nearly all the mathematical (dynamical) models of biological phenomena, the variables are positive, because they are numbers of species, concentrations of products,...

With respect to this constraint, two attitudes are possible:

- the mathematical model is whatever, and we decide that only the positive portions of the trajectories are meaningful.

- the mathematical model is built in such a way that the variables stay positive if the initial conditions are positive. 
We will study here the mathematical implications of the second choice, that seems to be more reasonable; but we don' t want to enter here into epistemological discussions. We will show that this hypothesis of positivity has often some important implications on the behaviour and the stability of the solutions of the system.

We will restrict ourselves to ordinary differential equations. For further references on the mathematical behaviour of biological models, see (2), (3) and (4).

\section{The linear case}

This case is not very interesting (there is often non-linearities in the biological models), but enables us to highlight the main ideas.

The model is:

$$
x^{\prime}=A x+b
$$

where $\mathrm{x}$ and $\mathrm{b}$ are two $\mathrm{n}$-dimensional vectors and $\mathrm{A}$ is $\mathrm{a} \mathrm{n}$ by $\mathrm{n}$ matrix. In the following, the inequality $x>0(x=0)$ means that $x_{i}>0\left(x_{i}=0\right)$ for each $i$.

The positivity hypothesis is that, if a solution of this equation starts from $x(0)=0$, it stays nonnegative for all time $t$. That means that the vector field along the faces $x_{1}=0$ of the positive orthant is inward. We must have:

$$
x_{i}=0 \Rightarrow x_{i}^{\prime}=0 \text { for all } x_{j}=0
$$

This condition gives us that $\mathrm{b}=0$ if we take $\mathrm{x}=0$. Then the condition is

$$
\sum_{i ? j} A_{i j} x_{j}+b_{i}=0 \text { for all } x_{j}=0
$$

and this implies that $A_{i j}=0$ for all $\mathrm{i}$ ? $\mathrm{j}$. Indeed, let us suppose that some $A_{i j}$ is negative, then it is possible to take a large $\mathrm{x}_{\mathrm{j}}$ to obtain a contradiction.

Therefore we obtain that the matrix A has to be off-diagonal nonnegative. This class of matrices has been extensively studied (1).

Until now, we made no assumptions about the existence of an equilibrium. Let us suppose that A is bijective: the equilibrium will be unique if it exists. 
Now, if we suppose that the equation

$$
\mathrm{Ax}=-\mathrm{b}
$$

admits a positive solution $\mathrm{x}^{*}$, we have that the image of a positive vector by an off diagonal nonnegative matrix is a negative vector, and a theorem (see (1) ) implies that A is stable ( its eigenvalues have a negative real part).

We can state the following proposition:

Proposition 1. Under the positivity hypothesis, the existence of a positive equilibrium implies the convergence of all the solutions towards this equilibrium for the linear systems.

\section{Lotka-Volterra models}

One of the most famous model in biology is the predator prey model of Lotka -Volterra, written: ( $\mathrm{x}$ is the prey and $\mathrm{y}$ the predator)

$$
\begin{aligned}
& x^{\prime}=a x-b x y \\
& y^{\prime}=c x y-d y
\end{aligned}
$$

the solutions are a family of closed curves in the positive quadrant of the phase space.

In the following, we consider the generalized Lotka-Volterra models in $\mathrm{n}$ dimensions, written (see (2)):

$$
x_{i}^{\prime}=x_{i}\left(\sum_{j=1}^{n} A_{i j} x_{j}+b_{i}\right) \quad i=1 \ldots n
$$

The variables $x_{i}$ are the number or concentrations of individuals, the $b_{i}$ is the growth rate of the species $i$ alone, and the terms $A_{i j}$ represent the interaction of the species $j$ on the growth of the species $i$; the sign of $A_{i j}$ depends on the interaction (predation , mutualism, competition, ...).

These systems are interesting to study from a mathematical point of view (2); they can have a very complex behaviour in dimension greater than two : limit cycles, chaos, strange attractors, ...

Concerning the positivity, the things seem to be simple: the faces $x_{i}=0$ are invariant, because if $x_{i}=0$, then $x_{i}^{\prime}=0$. Therefore (by a theorem of uniqueness of the solution of a differential equation) the interior of the positive orthant is also invariant. A solution that starts positive will stay positive. The structure of the Lotka-Volterra models implies that the hypothesis of positivity is verified. 
But it is always possible for a trajectory to go very close to the faces. What is then the biological meaning of the model for a variable near zero ? At a small scale, the population is rather discrete, and the modelling by a differential equation is doubtful. Moreover, a good model is often valid only at a given space scale, and has to be changed for small scales of the variables.

It is certain that this problem is a difficult one, and has no simple answer. To clarify the situation, we are going to assume a "strong positivity" hypothesis, i.e. we suppose that there is a given threshold , S, under which it is forbidden to fall. Of course, it is a strong hypothesis, that means that we impose, a priori, to each variable to stay away from zero. To simplify, we take the same threshold for all the variables.

A possible mathematical translation is the following (to simplify, we impose strict inequalities): on the hyperplane $x_{i}=S$, the field must be inward $\left(x_{i}^{\prime}>0\right)$ if the other $x_{j}$ are above the threshold, that gives the equation:

$$
S\left(A_{i i} S+\sum_{j} A_{i j} x_{j}+b_{i}\right)>0 \text { for all } x_{j}=S
$$

that implies, by the same reasoning as in the linear case, $A_{i j}=0$. The matrix $A$ has to be offdiagonal nonnegative. This property means that the Lotka-Volterra system is cooperative ( see (5) for a survey of cooperative systems) and that the flow respects the partial ordering of $\mathrm{R}^{\mathrm{n}}$. Such systems have very strong properties: under technical additional hypotheses (the matrix A has to be irreducible, i.e. its directed graph has to be strongly connected), then almost all bounded trajectory converge towards the set of equilibria. We will call this kind of behaviour "regular": in particular, there is no stable limit cycle or recurrent solution or chaos ..., but the solution can be unbounded.

Proposition 2. The hypothesis of strong positivity for the Lotka-Volterra system implies a regular behaviour of the solutions.

Let us now examine the equilibria. We make the usual assumption that the matrix $\mathrm{A}$ is bijective; the positive equilibrium will be unique if it exists, because it verifies:

$$
\mathrm{Ax}^{*}+\mathrm{b}=0
$$

Let us suppose that $x^{*}>\mathrm{S} \mathbf{1}$ ( $\mathbf{1}$ is the vector $(1,1, \ldots, 1)$ ), i.e. that the equilibrium is above the threshold.

It is easy then to use the geometrical properties of cooperative systems to build a ndimensional rectangle around the equilibrium; this rectangle will be invariant and contracting 
towards the equilibrium. The lower left corner ( the smallest point of the rectangle for the partial ordering) is the point $(\mathrm{S}, \mathrm{S}, \ldots, \mathrm{S})$; by hypothesis, the vector field is positive at this point $\left(\mathrm{A}\left(\mathrm{S} 1-\mathrm{X}^{*}\right)>0\right)$. The largest point can be taken as large as we want: take the point $\mathrm{y}$ such that $\mathrm{y}-\mathrm{x}^{*}=\mathrm{u}\left(\mathrm{x}^{*}-\mathrm{S} \mathbf{1}\right)$, where $\mathrm{u}$ is real positive; then the field at this point is

$$
\mathrm{A}\left(\mathrm{y}-\mathrm{x}^{*}\right)=\mathrm{u} \mathrm{A}\left(\mathrm{x}^{*}-\mathrm{S} \mathbf{1}\right)<0
$$

Therefore, by a well-known theorem (5), we obtain the global stability of the equilibrium in the region $\mathrm{x}_{\mathrm{i}}=\mathrm{S}$.

Proposition 3. Under the strong positivity hypothesis, the existence of a positive equilibrium above the threshold implies the global convergence of all the solutions towards this equilibrium for the Lotka-Volterra systems.

Let us remark that the result "A off diagonal positive" means that, from a biological point of view, all the interactions between species are mutualistic.

\section{A weaker positivity hypothesis}

We will try now to weaken a little bit this strong positivity hypothesis: it is easy to see that the results $A_{i j}=0$ comes from equation (1) because the region $x_{i}=S$ is unbounded. We make a weaker hypothesis by imposing that the solutions stay above a threshold $\mathrm{m}$ and below a threshold M; we choose still the same threshold for all the variables. We call this hypothesis the weak positivity hypothesis.

The mathematical translation is that the vector field on the n-dimensional rectangle of lower corner $(\mathrm{m}, \mathrm{m}, \ldots, \mathrm{m})$ and of upper corner $(\mathrm{M}, \mathrm{M}, . ., \mathrm{M})$ is (strictly) inward. Let us remark that it implies that there exists a positive equilibrium, by a simple argument of positively invariant domains. We suppose that $\mathrm{A}$ is still bijective, so this equilibrium is unique.

Let us write that the minimum of $x_{i}^{\prime}$ is positive on the face $x_{i}=m$, the other $x_{j}$ being inside the rectangle. To simplify, we suppose that the equilibrium is the vector

$(1,1, \ldots, 1)=\mathbf{1}$ (this hypothesis is consistent with the choice of the same threshold for the variables; moreover, in a Lotka-Volterra system, the equilibrium can always be scaled to $\mathbf{1}$ by a change of variables $y_{i}=x_{i} / x_{i}$ ). We have therefore:

$$
\sum_{\mathrm{j} ? \mathrm{i}} \mathrm{A}_{\mathrm{ij}}^{+}(\mathrm{m}-1)+\sum_{\mathrm{j} ? \mathrm{i}} \mathrm{A}_{\mathrm{ij}}^{-}(\mathrm{M}-1)+\mathrm{A}_{\mathrm{ii}}(\mathrm{m}-1)=0
$$

where $\mathrm{A}^{+}$is $\max (\mathrm{A}, 0)$ and $\mathrm{A}^{-}$is $\min (\mathrm{A}, 0)$. 
If we write now that the maximum of $x_{i}^{\prime}$ is negative on the face $x_{i}=M$, the other $x_{j}$ being in the rectangle, we obtain that:

$$
\sum_{j ? i} A_{i j}^{+}(M-1)+\sum_{j ? i} A_{i j}^{-}(m-1)+A_{i i l}(M-1)=0
$$

Then we can obtain a necessary condition by subtracting these two equations; if we denote by B the matrix such that

$$
B_{i i}=A_{i i} \text { for } i=1, \ldots n \text { and } B_{i j}=\left|A_{i j}\right| \text { for } i, j=1, \ldots ., n \text { i? } j
$$

(B is the matrix obtained by taking the absolute values of the off-diagonal elements of the matrix $\mathrm{A})$, then

$$
\mathrm{B}(\mathrm{M}-\mathrm{m}) \mathbf{1}=0
$$

or, because $\mathrm{M}>\mathrm{m}$

$$
\mathrm{B} 1=0
$$

that implies that $\mathrm{A}_{\mathrm{ii}}=0$ and that the absolute values of the off-diagonal elements of $\mathrm{A}$ are "not too strong" relatively to the negative diagonal.

But we are able to characterise more precisely these rectangles; let us denote by $\mathrm{C}$ the matrix such that

$$
C_{i j}=A_{i j} \text { if } A_{i j}=0 \text { and } 0 \text { if not, for } i, j=1, \ldots, n, i ? j \text { and } C_{i i}=0
$$

( $\mathrm{C}$ is a negative matrix built with the negative off-diagonal part of $\mathrm{A}$ ) then it is easy to see that the two above inequalities can be rewritten

$$
\begin{aligned}
& (\mathrm{m}-1) \mathrm{B} \mathbf{1}+(\mathrm{M}+\mathrm{m}-2) \mathrm{C} \mathbf{1}=0 \\
& (\mathrm{M}-1) \mathrm{B} \mathbf{1}+(\mathrm{M}+\mathrm{m}-2) \mathrm{C} \mathbf{1}=0
\end{aligned}
$$

but we know that $\mathrm{Bl}=\mathrm{u}<0$ ( we suppose to simplify that the inequality is strict), and, because $\mathrm{C}$ is negative, $\mathrm{Cl}=\mathrm{v}=0$. Moreover, let $\mathrm{r}=\frac{\mathrm{M}-1}{1-\mathrm{m}}>0$ because of the hypotheses. Then a little bit of calculus on these inequalities gives

$$
(\mathrm{u}+\mathrm{v})=\mathrm{r} \mathrm{v}
$$




$$
(\mathrm{u}+\mathrm{v}) \mathrm{r}=\mathrm{v}
$$

We try to maximize the dimension of the rectangle, that means that we maximize $r$. The solution have to verify:

$$
r=\left(1+\frac{u_{i}}{v_{i}}\right) \text { for each } i \text { such that } v_{i}<0
$$

and the best choice for $r$ is:

$$
r=\operatorname{Min}_{i}\left(1+\frac{u_{i}}{v_{i}}\right) \text { for each } i \text { such that } v_{i}<0
$$

What are the implications of this ? If $\mathrm{v}=0$, then the system is cooperative, and the choice of the size of the rectangle is free (as we have already seen). If $v$ ?0, then there are some negative elements in the matrix A, and the size of the rectangle is bounded; that is, for some choice of the minimum $\mathrm{m}$, the maximum $\mathrm{M}$ cannot be greater than some number (related to $\mathrm{r}$ ).

Proposition 4. To verify the weak positivity hypothesis, it is necessary that the two corners of the rectangle are related by the above inequality.

What happens now in this rectangle ? In fact, it is invariant (by hypothesis) and moreover contracting towards the equilibrium $\mathbf{1}$. To see that, let us consider the function:

$$
\mathrm{V}(\mathrm{x})=\operatorname{Max}_{\mathbf{i}}\left|\mathrm{x}_{\mathrm{i}}-1\right|
$$

and let us prove that this positive function decreases along the trajectories. If $\mathrm{k}$ is the subscript such that, at time $t,\left|x_{k}-1\right|$ is the maximum and $x_{k}>1$, then the sign of the time derivative $\mathrm{x}^{\prime} \mathrm{k}$ is the same that the sign of the expression

$$
\sum_{j=1}^{n} A_{k j}\left(x_{j}-1\right)
$$

and we can majorize the $\left(x_{j}-1\right)$ by $\left(x_{k}-1\right)$ and minorize the $\left(x_{j}-1\right)$ by $\left(1-x_{k}\right)$. We obtain that the above expression is smaller that $\mathrm{B}\left(\mathrm{x}_{\mathrm{k}}-1\right) \mathbf{1}$ which is negative because $\mathrm{Bl}$ is. Thus $\mathrm{x}_{\mathrm{k}}^{\prime}$ decreases. If $x_{k}<1$, we show similarly that $x^{\prime}{ }_{k}$ increases. Let us remark that it would be also true for any rectangle around the equilibrium.

Because $\mathrm{V}(\mathrm{x})$ is zero only if $\mathrm{x}=\mathbf{1}$, the family of rectangles decrease until the equilibrium. Thus we have shown that the region inside the rectangle is asymptotically stable. In fact, V is a Lyapunov function. We can state: 
Proposition 5. The weak positivity hypothesis (thresholds from above and from below) implies that the system is asymptotically stable in the whole region defined by the thresholds.

The proofs above show in fact that if one "centered rectangle" (a rectangle around $\mathbf{1}$ with the lower and the upper corners on the straight line $\lambda \mathbf{1})$ is strictly invariant, then $\mathrm{B} \mathbf{1}<0$, and therefore any centered rectangle will contract towards the equilibrium; the equilibrium is stable in the whole orthant. By contraposition, we obtain the

Proposition 6. If the equilibrium of the system is not globally asymptotically stable in the positive orthant, then there exists trajectories that leave any centered rectangle .

This weak positivity hypothesis seems to be biologically more reasonable. We can deduce from the above propositions some biological interpretations.

\section{Biological interpretation 1.}

To verify the weak positivity hypothesis, the self-interactions have to be negative, and the interactions between the species have to be "small" with respect to the self interactions. The individual rates of growth have to be positive.

One can compare these results to the well-known facts in dimension 2 (cf. (4, pp. 78-85)) : for a competitive Lotka-Volterra system, there is no extinction only if the interactions are small with respect to the individual growths. For a mutualistic Lotka-Volterra model, there is no explosion only if the same property is verified.

\section{Biological interpretation 2.}

It is not possible to choose the minimum and maximum thresholds independently; the model has to be defined for a given space scale, and the equilibrium is asymptotically stable in this region.

Maybe the more interesting is the contraposition of the result of convergence; let us suppose that the biologist is able to know that the equilibrium is not globally stable, because he observes a periodic trajectory for example.

\section{Biological interpretation 3.}

If the equilibrium is not globally stable, then there exists trajectories where some variables become very small or very large (smaller or larger than any given threshold). 
That means that it is not possible to find a space scale including all the trajectories; some of these trajectories are "dangerous" and could lead to an extinction or an explosion; moreover, it is not clear that the given model is able to modelize these trajectories : very often, the biological model must be changed if the species is in very small quantity ; there are additional phenomena like refuge, stochasticity, ... These trajectories have to be carefully inspected from a biological point of view.

Acknowledgment. The author thanks the " comite MMT, programme Environnement, CNRS ", for its support.

\section{References}

<1> A. Berman and R.J. Plemmons, "Nonnegative matrices in the mathematical sciences", Academic Press (1979)

<2> J. Hofbauer and K. Sigmund, "The theory of evolution and dynamical systems", Cambridge University Press (1988)

<3> J.D. Lebreton et C. Millier, "Modèles dynamiques déterministes en biologie", Masson $(1982)$

$<4>$ J.D. Murray, "Mathematical biology", Springer (1990)

<5> H.L. Smith, Systems of ordinary differential equations which generates an order preserving flow, SIAM Review, 30, pp. 87-113 (1988) 